\title{
Correction: Diagnostic Accuracy of Chest Computed Tomography Scans for Suspected Patients With COVID-19: Receiver Operating Characteristic Curve Analysis
}

\author{
Lianpin $\mathrm{Wu}^{1}$, MD; Qike Jin ${ }^{1}$, MD; Jie Chen ${ }^{2}$, MD, PhD; Jiawei He ${ }^{1}$, MD; David M Brett-Major ${ }^{3}$, MD; Jianghu James
} Dong $^{4}, \mathrm{PhD}$

${ }^{1}$ Department of Cardiology, the Second Affiliated Hospital \& Yuying Children's Hospital of Wenzhou Medical University, Wenzhou, China

${ }^{2}$ College of Optometry, Wenzhou Medical University, Wenzhou, China

${ }^{3}$ Department of Epidemiology, University of Nebraska Medical Center, Omaha, NE, United States

${ }^{4}$ Department of Biostatistics and Department of Medicine, University of Nebraska Medical Center, Omaha, NE, United States

Corresponding Author:

Jianghu James Dong, PhD

Department of Biostatistics and Department of Medicine

University of Nebraska Medical Center

984375 Nebraska Medical Center

Omaha, NE, 68198-4375

United States

Phone: 14025591976

Email: jianghu.dong@unmc.edu

\section{Related Article:}

Correction of: https://publichealth.jmir.org/2020/4/e19424/

(JMIR Public Health Surveill 2020;6(4):e25829) doi: 10.2196/25829

In "Diagnostic Accuracy of Chest Computed Tomography Scans for Suspected Patients With COVID-19: Receiver Operating Characteristic Curve Analysis" (JMIR Public Health Surveill 2020;6(4):e19424), the authors noted one error.

The affiliation for authors Lianpin Wu, Qike Jin, and Jiawei He was incorrectly listed as:

Department of Cardiology, Wenzhou Medical University, Wenzhou, China

The affiliation for these authors has been corrected to:
Department of Cardiology, the Second Affiliated Hospital \& Yuying Children's Hospital of Wenzhou Medical University, Wenzhou, China

The correction will appear in the online version of the paper on the JMIR Publications website on November 20, 2020, together with the publication of this correction notice. Because this was made after submission to PubMed, PubMed Central, and other full-text repositories, the corrected article has also been resubmitted to those repositories.

This is a non-peer-reviewed article. Submitted 17.11.20; accepted 17.11.20; published 20.11.20.

Please cite as:

Wu L, Jin Q, Chen J, He J, Brett-Major DM, Dong JJ

Correction: Diagnostic Accuracy of Chest Computed Tomography Scans for Suspected Patients With COVID-19: Receiver Operating Characteristic Curve Analysis

JMIR Public Health Surveill 2020;6(4):e25829

URL: http://publichealth.jmir.org/2020/4/e25829/

doi: $\underline{10.2196 / 25829}$

PMID: 33216725

CLianpin Wu, Qike Jin, Jie Chen, Jiawei He, David M Brett-Major, Jianghu James Dong. Originally published in JMIR Public Health and Surveillance (http://publichealth.jmir.org), 20.11.2020. This is an open-access article distributed under the terms of the Creative Commons Attribution License (https://creativecommons.org/licenses/by/4.0/), which permits unrestricted use, 
distribution, and reproduction in any medium, provided the original work, first published in JMIR Public Health and Surveillance, is properly cited. The complete bibliographic information, a link to the original publication on http://publichealth.jmir.org, as well as this copyright and license information must be included. 